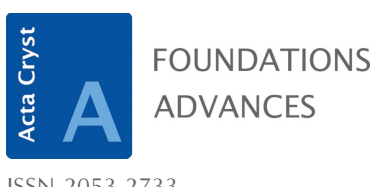

ISSN 2053-2733

Received 11 August 2015

Accepted 12 August 2015

Keywords: systematic absences; powder autoindexing; algorithm

\section{Distribution rules of systematic absences on the Conway topograph and their application to powder auto-indexing: erratum}

Ryoko Oishi-Tomiyasu*

High Energy Accelerator Research Organization, Tsukuba, Ibaraki, Japan. *Correspondence e-mail:
ryoko.tomiyasu@kek.jp

A correction is made to the article by Oishi-Tomiyasu [Acta Cryst. (2013), A69, 603-610].

Section 2 of the article by Oishi-Tomiyasu (2013) starts with the following sentence: 'Any crystallographic group $G$ is represented as the semidirect $R_{G} \ltimes L$, where $R_{G}$ is a point group of $G$ and $L$ is the lattice ...'. The phrase 'semidirect $R_{G} \times L$ ' should be replaced by 'group extension of $L$ by $R_{G}$ '. This mistake does not affect the other parts of the article.

\section{References}

Oishi-Tomiyasu, R. (2013). Acta Cryst. A69, 603-610. 